Surveillance

\title{
Welche Indikatoren eignen sich zur Beurteilung der MRSA-Situation?
}

\author{
P. Gastmeier', I. Chaberny' , D. Sohr', F. Schwab², E. Meyer', F. Daschner', H. Rüden² \\ 1'Institut für Medizinische Mikrobiologie und Krankenhaushygiene, Medizinische Hochschule Hannover \\ (Leiter: Prof. Dr. S. Suerbaum) \\ 2Institut für Hygiene, Charité, Universitätsmedizin Berlin \\ (Leiter: Prof. Dr. H. Rüden) \\ 3Institut für Umweltmedizin und Krankenhaushygiene, Universitätsklinikum Freiburg \\ (Leiter: Prof. Dr. F. Daschner)
}

klinikarzt 2004; $33(1+2): 10-14$

Die Inzidenz der MRSA-Infektionen in Deutschland steigt kontinuierlich. Eine Voraussetzung für die Durchsetzung geeigneter und kosteneffektiver Präventionsmaßnahmen ist die Überwachung der MRSA-Fälle (methicillinresistenter Staphylococcus aureus) und das regelmäßige Feedback der Daten an die Stationen. Deshalb fordert das Infektionsschutzgesetz die regelmäßige Aufzeichnung von multiresistenten Erregern. Allerdings besteht keine Übereinkunft darüber, welche Raten zur Kennzeichnung der MRSA-Situation einer Station oder eines Krankenhauses berechnet werden sollten, um sie als Basis für Vergleiche zwischen verschiedenen Stationen und Krankenhäusern zu benutzen. Mithilfe der MRSA-Daten von 20 Intensivstationen des Krankenhaus-Infektions-Surveillance-Systems (KISS) und der MRSA-Daten derselben Intensivstationen des Surveillance-Systems SARI (Surveillance der Antibiotika-Anwendung und der bakteriellen Resistenzen auf Intensivstation) versuchen wir hier, die Eignung verschiedener möglicher Raten zu untersuchen, um daraus Empfehlungen für die Surveillance abzuleiten.

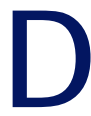
as Problem resistenter Bakterienstämme - wie zum Beispiel die methicillinresistenten Staphylococcus-aureus-Stämme (MRSA) - wird auch in Deutschland immer größer. Während beispielsweise in deutschen Intensivstationen 1997 nur 8\% aller Staphylococcus-aureus-bedingten nosokomialen Infektionen durch MRSA hervorgerufen wurden, waren es 2002 bereits $26,9 \%$. Für nosokomiale Pneumonien betrug der MRSA-Anteil $24,4 \%$, für die nosokomiale primäre Sepsis sogar 35,2\%. Das hat zur Folge, dass die therapeutischen Optionen immer mehr eingeschränkt werden, oder dass häufig nur noch wenige Antibiotika zur Verfügung stehen, die wirksam sind und zudem die nötige Bioverfügbarkeit aufweisen, um in den betroffenen Organsyste- men entsprechende Wirkstoffspiegel zu erreichen. So steigen die Therapiekosten, und die Nebenwirkungen der Antibiotika-Anwendung nehmen zu.

Allein die kontinuierliche Erfassung nosokomialer Infektionen und der Resistenzprobleme und ein entsprechendes regelmäßiges Feedback kann jedoch bekanntermaßen die Situation verbessern (5). Deshalb sind seit Januar 2001 alle Krankenhäuser und ambulanten Einrichtungen entsprechend $\S 23$ des Infektionsschutzgesetzes verpflichtet, Erreger mit speziellen Resistenzen und Multiresistenzen aufzuzeichnen und zu bewerten. Dadurch ist jedoch nicht nur eine Analyse der eigenen Daten über die Zeit hinweg möglich, viel interessanter ist der Vergleich $\mathrm{zu}$ anderen Krankenhäusern und
Abteilungen. Jedoch wie soll diese Bewertung erfolgen?

- Sollen nur nosokomiale MRSAFälle oder sämtliche beobachteten Fälle berücksichtigt werden?

- Soll sich die Analyse nur auf Infektionen oder gleichermaßen auf Kolonisationen konzentrieren?

- Auf welche Grundgesamtheiten soll man die identifizierten multiresistenten Erreger beziehen, auf entsprechende S.-aureus-Fälle oder besser auf 1000 Patiententage?

- An welchen Daten kann man sich orientieren?

Der folgende Artikel untersucht am Beispiel der MRSA auf deutschen Intensivstationen, wie man vorgehen kann, woran man sich orientieren sollte und welche offenen Fragen existieren.

\section{Methode}

In Deutschland erfassen zwei Datenbanken regelmäßig Daten zur MRSA-Situation auf Intensivstationen: So liefert das KrankenhausInfektions-Surveillance-System des Nationalen Referenzzentrums für Surveillance von nosokomialen Infektionen (KISS) inzwischen seit 1997 regelmäßig Informationen über die Situation auf Intensivstationen. Bis Ende 2002 haben 274 der 
etwa 1700 deutschen Intensivstationen beatmungsassoziierte Pneumonien und Bronchitiden, zentralvenenkatheterassoziierte SepsisFälle und harnwegkatheterassoziierte Harnwegsinfektionen sowie ihre Erregersituation und ausgewählte Schlüsselresistenzen (darunter MRSA) nach einheitlichen Definitionen und Erfassungsmodalitäten an KISS gesendet. Damit existiert ein guter Überblick über die durchschnittlichen nosokomialen Infektionsraten und ihre Verteilung (3).

Die jeweils aktuellen, nach Art der Intensivstation stratifizierten Daten können auf der Homepage des Nationalen Referenzzentrums (NRZ) für die Surveillance von nosokomialen Infektionen eingesehen werden (http://www.nrz-hygiene. de). Zusätzlich besteht seit einiger Zeit die Möglichkeit, Orientierungsdaten zur Anwendung von Antibiotika und zur Resistenzsituation einzusehen.

Das Institut für Umweltmedizin und Krankenhaushygiene des Universitätsklinikums Freiburg hat im Jahr 2000 begonnen, parallel zur
KISS-Datenbank eine zweite Datenbank aufzubauen: SARI (,Surveillance der Antibiotika-Anwendung und der bakteriellen Resistenzen auf Intensivstationen“) erfasst inzwischen standardisierte Daten zur Anwendung von Antibiotika und zur Resistenzsituation aus 35 bundesweit verteilten KISS-Intensivstationen (http://www.sari-antibiotika. de).

Mithilfe der SARI-Daten können Sie beispielsweise überprüfen, ob ein bestimmtes Antibiotikum in der eigenen Intensivstation wesentlich häufiger als in anderen Intensivstationen verwendet wird. Diese Information kann eine wichtige Hilfestellung für die Veränderung von Therapiegewohnheiten sein. Zusätzlich kann man sich hinsichtlich des Vorkommens bestimmter „Schlüssel“Resistenzen orientieren und daraus Schlussfolgerungen für Therapieveränderungen oder die Modifikation von Maßnahmen zur Erregerausbreitung auf der eigenen Intensivstation ableiten (3).

Zur Beantwortung der Fragestellung wurden die Daten der SARI-
Intensivstationen zum Vorkommen von MRSA herangezogen, und es wurden für denselben Zeitraum die KISS-Daten dieser Stationen zum Vorkommen von nosokomialen MRSA-Infektionen benutzt. Der Beobachtungszeitraum war von Februar 2000 bis Dezember 2002 - allerdings haben einige Intensivstationen erst später begonnen, Daten an SARI zu senden.

In diesen Fällen wurde selbstverständlich auch aus den KISS-Daten nur dieser Zeitraum für den Vergleich herangezogen. In KISS wird eine nosokomiale MRSA-Infektion dann erfasst, wenn die CDC-Definitionen für eine nosokomiale Infektion auf der Intensivstation erfüllt sind (2). SARI erfasst MRSA-Isolate auch dann, wenn es sich um Kolonisationen oder um bereits bei Aufnahme auf die Intensivstation vorliegende Infektionen handelt. Persistiert der MRSA-Nachweis über längere Zeit, geht jeder Stamm eines Patienten einmal pro Monat in die Datenbank ein (7).

In den an KISS und SARI teilnehmenden Intensivstationen werden

Tab. 1 KISS- und SARI-Daten aus 20 ausgewählten Intensivstationen

\begin{tabular}{|c|c|c|c|c|c|c|c|c|c|}
\hline \multirow[t]{2}{*}{$\begin{array}{l}\text { Intensiv- } \\
\text { station }\end{array}$} & \multirow[t]{2}{*}{$\begin{array}{l}\text { Patienten- } \\
\text { tage }\end{array}$} & \multicolumn{2}{|c|}{$\begin{array}{l}\text { nosokomiale } \\
\text { Infektionen } \\
\text { nach KISS }\end{array}$} & \multicolumn{2}{|c|}{$\begin{array}{l}\text { Isolate nach SARI } \\
\text { (Kolonisation und } \\
\text { Infektionen) }\end{array}$} & \multicolumn{2}{|c|}{$\begin{array}{l}\text { MRSA pro } \\
1000 \text { Patienten- } \\
\text { tage }\end{array}$} & \multicolumn{2}{|c|}{$\begin{array}{l}\text { MRSA/ } \\
\text { S. aureus (\%) }\end{array}$} \\
\hline & & S. aureus & MRSA & S. aureus & MRSA & KISS & SARI & KISS & SARI \\
\hline 1 & 12179 & 12 & 2 & 148 & 21 & 0,2 & 1,7 & 17 & 14 \\
\hline 2 & 12694 & 10 & 2 & 104 & 27 & 0,2 & 2,1 & 20 & 26 \\
\hline 3 & 8024 & 11 & 3 & 101 & 14 & 0,4 & 1,7 & 27 & 14 \\
\hline 4 & 6662 & 10 & 2 & 88 & 11 & 0,3 & 1,7 & 20 & 12 \\
\hline 5 & 11598 & 44 & 19 & 361 & 107 & 1,6 & 9,2 & 43 & 30 \\
\hline 6 & 9330 & 13 & 4 & 142 & 49 & 0,4 & 5,3 & 31 & 34 \\
\hline 7 & 8496 & 21 & 8 & 229 & 76 & 0,9 & 8,9 & 38 & 33 \\
\hline 8 & 9771 & 16 & 10 & 256 & 105 & 1,0 & 10,7 & 62 & 41 \\
\hline 9 & 14384 & 60 & 45 & 627 & 251 & 3,1 & 17,4 & 75 & 40 \\
\hline 10 & 21435 & 86 & 7 & 561 & 88 & 0,3 & 4,1 & 8 & 16 \\
\hline 11 & 13218 & 29 & 19 & 465 & 197 & 1,4 & 14,9 & 65 & 42 \\
\hline 12 & 17521 & 22 & 0 & 126 & 9 & 0 & 0,5 & 0 & 7 \\
\hline 13 & 7827 & 11 & 5 & 91 & 15 & 0,6 & 1,9 & 46 & 16 \\
\hline 14 & 7202 & 15 & 0 & 145 & 1 & 0 & 0,1 & 0 & 1 \\
\hline 15 & 6009 & 13 & 1 & 605 & 39 & 0,2 & 6,5 & 8 & 6 \\
\hline 16 & 21521 & 42 & 8 & 350 & 80 & 0,4 & 3,7 & 19 & 23 \\
\hline 17 & 11466 & 47 & 5 & 295 & 19 & 0,4 & 1,7 & 11 & 6 \\
\hline 18 & 7165 & 25 & 4 & 115 & 20 & 0,6 & 2,8 & 16 & 17 \\
\hline 19 & 5316 & 32 & 5 & 121 & 19 & 0,9 & 3,6 & 16 & 16 \\
\hline 20 & 9559 & 10 & 0 & 117 & 14 & 0 & 1,5 & 0 & 12 \\
\hline Summe & 221377 & 529 & 149 & 5047 & 1162 & 13,0 & 100,1 & 521,6 & 408,1 \\
\hline Mittelwert & & & & & & 0,67 & 5,2 & 28,2 & 23,0 \\
\hline
\end{tabular}




\section{Tab. 2 Vergleich der Gesamtdaten der Studien-Intensivstationen $(\mathbf{n}=\mathbf{2 0})$ mit der Gesamtmenge der KISS-Intensivstationen}

\begin{tabular}{|c|c|c|}
\hline & $\begin{array}{l}\text { Studien-Intensivstationen } \\
\qquad(\mathrm{n}=20) \\
\text { (Februar 2000 bis } \\
\text { Dezember 2002) }\end{array}$ & $\begin{array}{l}\text { alle KISS-Intensivstationen } \\
(\mathrm{n}=274) \\
\text { (Januar 1997 bis } \\
\text { Dezember 2002) }\end{array}$ \\
\hline Patiententage & 221377 & 2145793 \\
\hline nosokomiale MRSA-Infektionen & 149 & 599 \\
\hline nosokomiale S.-aureus-Infektionen & 529 & 2989 \\
\hline MRSA/S. aureus (\%) & 28,2 & 20 \\
\hline MRSA pro 1000 Patiententage & 0,67 & 0,28 \\
\hline
\end{tabular}

unterschiedliche mikrobiologische Methoden zur Klassifikation der MRSA angewandt. Zur Festlegung der Oxacillin-Resistenz erfolgt teilweise eine Orientierung an DIN, teilweise an NCCLS („National Committee for Clinical Laboratory Standards"). Für die vorliegende Analyse wurde dies nicht berücksichtigt.

Von den 35 Intensivstationen, die an SARI und KISS teilnehmen, beobachteten innerhalb des Zeitraumes von 35 Monaten nur 20 zehn oder mehr nosokomiale S.-aureusInfektionen. Um zufällige Einflüsse möglichst weit ausschließen zu können, wurden die Analysen deshalb auf diese 20 Intensivstationen begrenzt. Folgende Anteile bzw. Raten zur Beurteilung der MRSA-Situation wurden für jede einzelne Intensivstation bestimmt (Tab. 2):

- nosokomiale MRSA-Infektionen pro 1000 Patiententage (KISS)

- nosokomiale MRSA-Infektionen pro nosokomiale S.-aureus-Infektionen in \% (KISS)

- MRSA-Isolate pro 1000 Patiententage (SARI)

- MRSA-Isolate pro S.-aureus-Isolate (jeweils nach Elimination von Copystrains) in \% (SARI).

Zusätzlich wurden für diese Anteile und Raten über die 20 in die Analyse einbezogenen Intensivstationen die gepoolten arithmetischen Mittel bestimmt.

\section{Ergebnisse}

Nach den KISS-Daten konnte bis Dezember 2002 bei 2989 nosokomialen Infektionen S. aureus als Erreger bestimmt werden. Damit war dieser Keim der häufigste nosokomiale Infektionserreger auf den In- tensivstationen (16,5\%), 26,9\% davon waren MRSA (4). Im Durchschnitt wurden im Jahr 2002 pro 1000 Patiententage 0,34 nosokomiale MRSAInfektionen beobachtet. Dabei gibt es eine große Variationsbreite zwischen den Intensivstationen. Von den insgesamt 228 im Jahr 2002 an KISS teilnehmenden Intensivstationen hatten 143 (62,7\%) keine einzige nosokomiale MRSA-Infektion, auf 44 Intensivstationen $(19,3 \%)$ waren jedoch mindestens $50 \%$ der nosokomialen S.-aureus-Infektionen durch MRSA bedingt.

Unter den für die weitere Analyse ausgewählten 20 Intensivstationen waren sieben interdisziplinäre, fünf chirurgische, vier internistische sowie vier neurochirurgische Intensivstationen. Insgesamt wurden in diesen Intensivstationen im Beobachtungszeitraum zwischen Februar 2000 und Dezember 2002221377 Patiententage erfasst. Dabei wurden nach KISS über 529 nosokomiale S.aureus-Infektionen berichtet - 149 davon waren MRSA-Infektionen $(28,2 \%)$. Im selben Zeitraum wurden im Rahmen von SARI 5047 S.aureus-Isolate erfasst, davon waren 1162 MRSA-Stämme (23,0\%). Tabelle 1 zeigt die Daten für die Intensivstationen.

Während der Beobachtungsperiode sind keine MRSA-Ausbruchsereignisse auf den 20 Intensivstationen beobachtet worden, in einigen dieser Stationen hat sich dagegen ein insgesamt hohes endemisches Niveau des MRSA-Vorkommens entwickelt. Nur in drei beteiligten Intensivstationen wurden keine nosokomialen MRSA-Infektionen gesehen. Die Verteilung der 20 Intensivstationen nach den vier verschiede- nen Endpunkten ist in den Abbildungen 1 und 2 dargestellt.

Ausgehend von der Vermutung, dass die Verteilung der Intensivstationen nach den MRSA pro 1000 Patiententage entsprechend den SARI-Daten die belastbarsten Informationen zur Beurteilung eines MRSA-Problems liefert (Abb. 2, links), wurden die vier Intensivstationen, welche die höchste Zahl von MRSA-Isolaten pro 1000 Patiententage liefern, rot und mit ihrer entsprechenden Nummer markiert. Diese „Ausreißer“ wurden weitgehend ebenso bei Anwendung der anderen verwendeten Untersuchungsendpunkte als „Ausreißer“ identifiziert. Ausnahme ist die Intensivstation 8. Sie schneidet in einer Darstellung der MRSA-Fälle pro $1000 \mathrm{~Pa}$ tiententage immer günstiger ab als wenn der prozentuale Anteil der MRSA/S.-aureus-Isolate zugrunde gelegt wird.

\section{Diskussion}

Die für die Analyse ausgewählte Anzahl von 20 Intensivstationen ist relativ klein und zeichnet sich im Vergleich zur Gesamtmenge der KISS-Intensivstationen durch eine deutlich höhere MRSA-Inzidenz aus. Für die Beantwortung der zu untersuchenden Frage kann das aber eher von Vorteil sein.

Wie bereits erwähnt, liefert die KISS-Datenbank nur Informationen zu MRSA und den nosokomialen Infektionen, diese sind natürlich nur die Spitze des Eisberges. Wesentlich mehr Intensivpatienten sind kolonisiert. Die Entwicklung von Resistenzproblemen in Intensivstationen folgt meist einem bestimmten Muster: Anfangs sind es nur wenige, sporadische Fälle - manchmal wird ein Ausbruch bemerkt. Erregerquellen in der Patientenumgebung oder die Aufnahme eines kolonisierten bzw. infizierten Patienten können solche initialen Ausbrüche hervorrufen. Im Laufe der Zeit nimmt die Inzidenz der Infektionen $\mathrm{zu}$, und ein beträchtlicher Teil der Patienten ist kolonisiert. Dann jedoch hat sich bereits eine endemische Situation entwickelt, und die Elimination dieser resistenten $\mathrm{Er}$ reger von der Station ist sehr schwer oder sogar praktisch unmöglich. 
Deshalb ist es für die Surveillance der MRSA-Situation sicher am besten, sich auf die Gesamtzahl der MRSA-Isolate nach Elimination von Copystrains (identische Isolate bei einem Patienten aus wiederholten Untersuchungsmaterialien) zu beziehen, wie es auch die Empfehlungen zur Durchführung der Surveillance nach $\S 23$ des Infektionsschutzgesetzes vorschlagen (6). Dabei muss dann aber sichergestellt sein, dass - ähnlich wie bei SARI Copystrains nicht berücksichtigt werden.

Ein möglicher Kritikpunkt ist außerdem, dass eine Ausrichtung der Surveillance auf die Endpunkte „MRSA-Isolate pro 1000 Patiententage“ oder „MRSA-Isolate pro S.-aureus-Isolate (\%)“ die unterschiedliche Untersuchungsdichte in verschiedenen Intensivstationen und das unterschiedliche Screeningverhalten im Umkreis von MRSA-Patienten nicht berücksichtigt. Zudem bekommen viele Intensivstationen eine hohe Anzahl von Patienten mit MRSA bei Aufnahme zugewiesen. Diese spielen zwar für das Management auf der Station eine Rolle, sollten aber nicht zur Beurteilung der Präventionsmaßnahmen herangezogen werden. Deshalb haben wir seit Jahresbeginn 2002 bei KISS für Intensivstationen und bei „deviceKISS“ begonnen, eine Modifikation der bisherigen MRSA-Surveillance zur Beobachtung der MRSA-Ausbreitung auf Intensivstationen einzuführen.

Erfasst werden jetzt nicht nur die nosokomialen Infektionen, sondern alle Fälle mit MRSA-Nachweis. Zudem wird zwischen bereits bei Aufnahme mitgebrachten und auf der Station erworbenen Fällen differenziert. Dafür gelten folgende Definitionen (KISS-Surveillance-Protokolle für Intensivstationen und für „device“-KISS):

\section{MRSA wurde mitgebracht}

Bei der Aufnahme des Patienten war die MRSA-Besiedlung entweder bereits bekannt oder der MRSAStamm aus Untersuchungsmaterial isoliert, das innerhalb von 48 Stunden nach der Aufnahme abgenommen wurde.

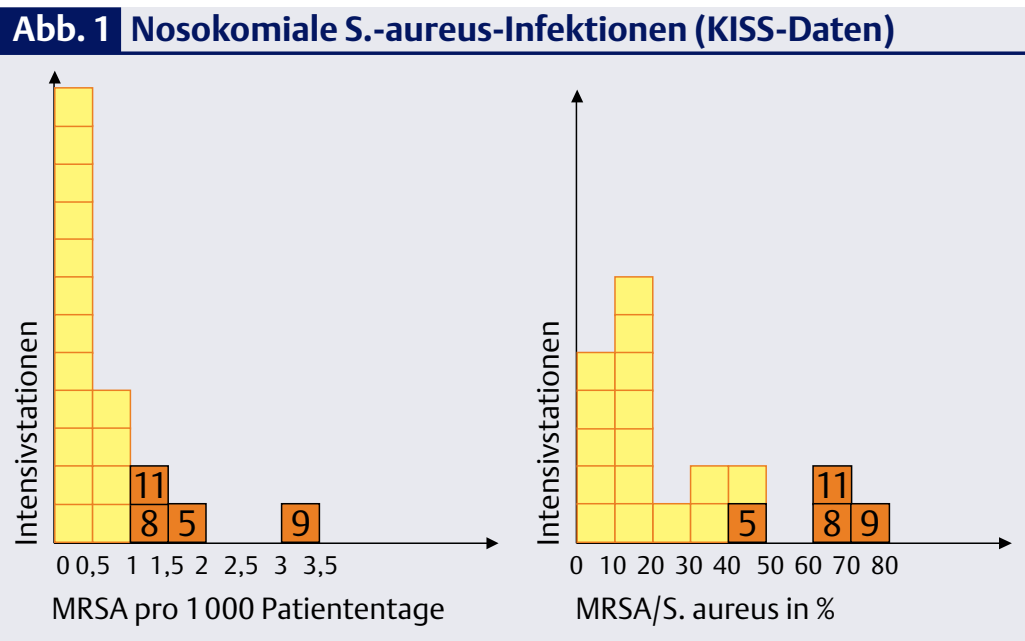

\section{Abb. 2 Erfasste S.-aureus-Isolate (SARI-Daten)}

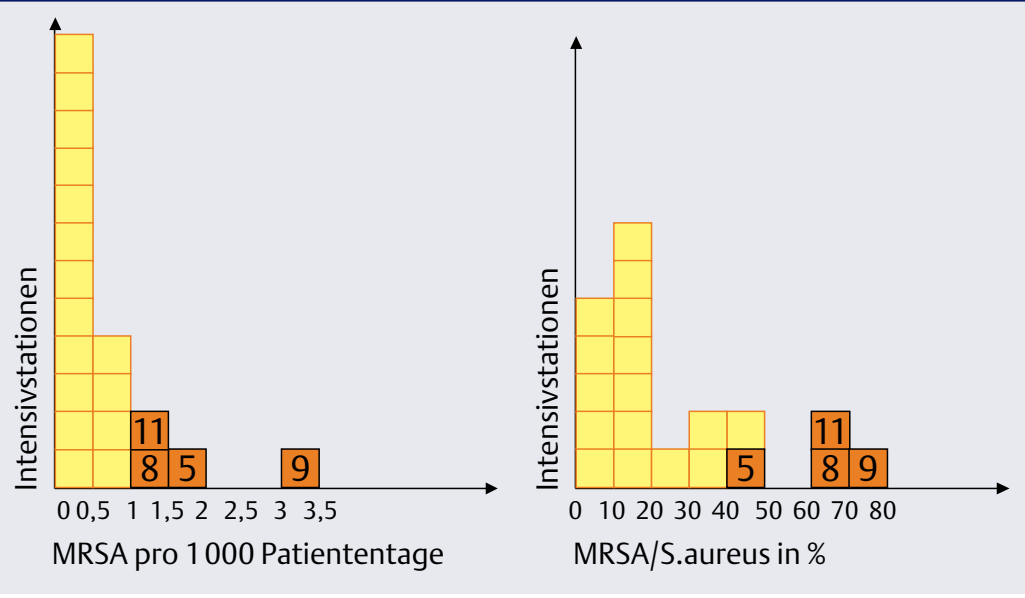

\section{MRSA wurde auf Station erworben}

Der MRSA-Stamm wurde aus einem während des Aufenthaltes auf dieser Station (nach den ersten 48 Stunden) abgenommenen Untersuchungsmaterial isoliert. Das gilt auch dann, wenn nicht ausgeschlossen werden kann, dass eine Besiedlung mit MRSA eventuell bereits bei Aufnahme vorlag, aber in den ersten 48 Stunden kein Untersuchungsmaterial abgenommen wurde und keine Nachweise von vorbehandelnden Krankenhäusern, Stationen oder Ärzten vorliegen.

Wie bei allen Surveillance-Definitionen gilt selbstverständlich auch hier, dass diese Definitionen nicht in jedem Einzelfall der wahren Situation entsprechen. Für die Surveillance ist es aber generell notwendig, einfach anwendbare, einheitliche Definitio- nen festzulegen. Zwar nimmt man bei deren Anwendung in Kauf, dass dadurch einige Infektionen zu viel und andere zu wenig gezählt werden, entscheidend ist jedoch, dass es einheitlich so gehandhabt wird.

Weiterhin sollte pro Station bzw. Abteilung erfasst werden, wie viel Kontaktpatienten von MRSA-Patienten untersucht wurden und bei welchem Anteil ein MRSA-Stamm nachgewiesen wurde. Insgesamt schlagen wir im Sinne der Vergleichbarkeit der Daten und in Weiterentwicklung der Empfehlungen des Robert-Koch-Instituts auf der Basis des Infektionsschutzgesetzes (6) folgendes Vorgehen für die Surveillance von MRSA vor:

(1) monatliche Erfassung aller MRSA-Fälle pro Station

(2) Differenzierung zwischen mitgebrachten und nosokomialen Fällen (siehe Definition oben) 


\section{Ins Netz gegangen}

www.cdc.gov/ncidod/diseases/index.htm

Informationen rund um Infektionskrankheiten finden Sie auf diesem Angebot des Centers of Disease Control (CDC). Stichworte zum Thema sind in einer alphabetisch geordneten Aufzählung zusammengefasst, jedem Stichwort sind natürlich ausführliche Informationen zugeordnet. Auch wenn Sie Auskünfte zum Thema Reisemedizin suchen, sind Sie auf dieser - natürlich englischsprachigen - Internetseite gut aufgehoben. Sie können sich sowohl über die verschiedenen Infektionskrankheiten, die in der Reisemedizin eine Rolle spielen, nötige Impfungen oder die Situation an Ihrem Reiseziel (z.B. Ausbrüche) informieren.

\section{www.dghm.org}

Nicht nur einen aktuellen Veranstaltungskalender und Stellenangebote finden Sie auf der Homepage der Deutschen Gesellschaft für Hygiene und Mikrobiologie. Lesen Sie hier, welche Auswirkungen die DRGs auf die Mikrobiologie, Infektiologie und Hygiene haben werden (Wir über uns / aktuelle Aktivitäten der DGHM). Auch wenn Sie auf der Suche nach aktuellen Leitlinien sind, sind Sie hier an der richtigen Adresse. Zudem finden Sie unter dieser Internetadresse auch die Nationalen Referenzzentren und Konsiliarlaboratorien.

\section{www.nrz-hygiene.de}

Sie suchen Informationen zur Situation nosokomialer Infektionen auf deutschen Intensivstationen? Dann sind Sie auf der Homepage des Nationalen Referenzzentrums (NRZ) für die Surveillance von nosokomialen Infektionen genau richtig. Denn in der KISS-Datenbank sind Daten von etwa 1700 deutschen Intensiv-

stationen zur Erregersituation gespeichert. Damit existiert ein guter Überblick über die nosokomialen Infektionen und ihre durchschnittliche Verteilung. Zusätzlich besteht seit einiger Zeit die Möglichkeit, Orientierungsdaten zur Anwendung von Antibiotika und zur Resistenzsituation einzusehen.

\section{www.sari-antibiotika.de}

Die SARI-Datenbank umfasst inzwischen standardisierte Daten zur Anwendung von Antibiotika und zur Resistenzsituation aus 35 bundesweit verteilten KISS-Intensivstationen. Hier finden Sie unter anderem Referenzdaten zur Antibiotikaanwendung - sogar stratifiziert nach den verschiedenen Intensivstationen. Sie können aber auch nachlesen, welche Leitlinien zur Antibiotikatherapie auf SARI-Intensivstationen eingehalten werden.

\section{www.zuendstoff-antibiotika-}

\section{resistenz.de/frame.html}

An Laien richtet sich die Initiative „Zündstoff Antibiotikaresistenz“. Hier wird allgemeinverständlich erklärt, wie Antibiotika wirken oder was es mit der Antibiotikaresistenz auf sich hat. Weiterführende Informationen finden Interessierte über die „Web-“ und „Buch-Tipps“ auf der Internetseite der Initiative.
(3) Differenzierung zwischen Kolonisationen und behandlungspflichtigen Infektionen

(4) jährliche Berechnung der Inzidenzdichte der MRSA-Fälle (pro 1000 Patiententage) pro Station

(5) jährliche Berechnung der Inzidenzdichte der nosokomialen MRSA-Infektionen (pro $1000 \mathrm{~Pa}$ tiententage)

(6) Bestimmung des Quotienten aus (5) und (4) als Maß für die Entwicklung von nosokomialen MRSA-Infektionen unter einem bestimmten Kolonisationsdruck

(7) Erfassung aller Untersuchungen von Kontaktpatienten sowie des Anteils derjenigen mit MRSANachweis

(8) Zusammenfassende Analysen für das Krankenhaus.

In den meisten Krankenhäusern wird diese Statistik sicherlich vom Hygienefachpersonal bearbeitet werden und in vielen Kliniken bereits in dieser oder ähnlicher Form schon verfügbar sein. Je mehr Krankenhäuser diese Daten in einheitlicher Weise erfassen, auswerten und miteinander vergleichen, desto eher wird es gelingen, Schwellenwerte zu generieren, die zu sofortigen Interventionsmaßnahmen führen sollten.

Natürlich müssen SurveillanceDaten Konsequenzen haben. Die Entwicklung der Daten der letzten Jahre zeigt, dass in Deutschland dringend Veränderungen im Umgang mit MRSA-Patienten nötig sind, wenn man die Situation noch beherrschen will. In einigen Intensivstationen scheint der Schwellenwert schon überschritten, bis zu dem man damit rechnen kann, dass sich die Situation wieder auf einen geringeren MRSA-Anteil reduzieren lässt.

Surveillance-Befunde sollen dazu führen, das bisherige Handeln zu überdenken und zu verändern. Es reicht nicht, entsprechende Leitlinien für den Umgang mit MRSA-Patienten zu haben. Die Compliance im Umgang damit muss regelmäßig überprüft werden. Teilweise ist auch zu überlegen, ob durch Einführung von Screeninguntersuchungen von bestimmten Risikopatienten bei Aufnahme auf die Intensivstation eine Verbesserung erreicht werden kann.
Lucet et al. haben über ihre guten Erfahrungen damit vor kurzem berichtet (6). Deshalb sollte eine Einigung auf einheitliche Surveillance-Methoden für die MRSA-Situation und entsprechende Schwellenwerte für das Erkennen von besonderen Problemen erfolgen. KISS und SARI können dazu sicher interessante Beträge leisten.

\section{Surveillance - Which Indicators are Appropriate for Assessing the MRSA Situation?}

The incidence of MRSA infections in German hospitals is increasing. Surveillance of MRSA cases and their routine feedback to the wards is a prerequisite for introducing appropriate and cost effective infection control measures. Therefore, prospective surveillance of multiresistant microorganisms is required by the German Protection against Infection Act. However, there is no agreement available, which rates should be used for characterising the MRSA situation of a given ward or hospital in order to use them for comparison between wards and hospitals. The data of 20 intensive care units participating in the Krankenhaus-Infektions-Surveillance-System (KISS) and in the surveillance system SARI (Surveillance System of Antibiotic Use and Bacterial Resistance in German Intensive Care Units) were used to investigate the appropriateness of various rates and recommendations for surveillance are given.

\section{Key Words}

surveillance - S. aureus - MRSA prevention

\section{Literatur bei der Redaktion / im Internet unter www.klinikarzt.info}

\section{Anschrift für die Verfasser}

Prof. Dr. Petra Gastmeier Institut für Medizinische Mikrobiologie und Krankenhaushygiene

Medizinische Hochschule Hannover Carl-Neuberg-Str. 1

30625 Hannover 